\title{
INCREASE IN EFFECTIVE FLUORESCENCE LIFETIME BY CROSS-RELAXATION EFFECT DEPENDING ON TEMPERATURE OF Nd/Cr:YAG CERAMIC USING WHITE-LIGHT PUMP SOURCE
}

\author{
TAKU SAIKI*\&, MASAHIRO NAKATSUKA ${ }^{\dagger}$, KANA FUJIOKA \\ SHINJI MOTOKOSHI ${ }^{\dagger}$, KAZUO IMASAKI ${ }^{\dagger}$ and YUKIO IIDA* \\ *Department of Electrical and Electronic Engineering \\ Faculty of Engineering Science, Kansai University \\ 3-3-35 Yamate-cho, Suita, Osaka 564-8680, Japan \\ $\dagger$ Institute for Laser Technology, 1-8-4 Utsubo-honmachi \\ Osaka, Osaka 550-0004, Japan \\ Institute of Laser Engineering, Osaka University, \\ 2-6 Yamada-oka, Suita, Osaka 565-0871, Japan \\ stsaiki@kansai-u.ac.jp
}

Received 10 January 2013

Revised 14 February 2013

Accepted 27 February 2013

Published 10 April 2013

\begin{abstract}
An increase in the effective fluorescence lifetime of $\mathrm{Nd} / \mathrm{Cr}$ :YAG ceramics with temperature dependence was observed. The dependence on the doped $\mathrm{Cr}$ ion density for the increased effective fluorescence lifetime was also investigated. The effective lifetime of fluorescence in the ceramics increased from 1.1 to $1.8 \mathrm{~ms}$ owing to the phonon-assist cross-relaxation induced by the excited Cr ions, which is commonly observed in Tm:YAG or glass lasers. The increase in effective fluorescence lifetime can be explained by spontaneous emissions occurring from the excited $\mathrm{Nd}$ ions and excitations of the $\mathrm{Nd}$ ions from the lower level to the upper level at the same time. Additionally, an experiment for laser oscillations with the temperature of the laser material controlled was performed, and a remarkable increase of the output laser energy owing to cross-relaxation was observed when the temperature increased. The obtained maximum laser energy output was near twice that without cross-relaxation. The ceramics are considered promising lamp- or solar-pumped solid-laser materials owing to the efficient laser action based on this cross-relaxation.
\end{abstract}

Keywords: Solar pumping; Nd/Cr:YAG ceramic; phonon-assisted cross-relaxation.

\section{Introduction}

Flash-lamp-pumped lasers as well as gas lasers are being used in the industry. Lasers have various applications. Here, the total efficiency is very important for reducing economical cost. Nd/Cr:YAG ceramic lasers with highly doped $\mathrm{Cr}^{3+}$ ions have been developed. ${ }^{1,2}$ The 
ceramic lasers are solid-state lasers pumped with lamp light. A high optical conversion efficiency of $40 \%$ has been achieved using a rod-type $\mathrm{Nd}^{3+} / \mathrm{Cr}^{3+}$ :YAG ceramic. ${ }^{3}$ Other reports on this laser material obtained by pumping lamp or solar light have been published. ${ }^{4,5}$ Solar-pumped lasers ${ }^{6,7}$ have also been studied. Such lasers are applicable to the generation of renewable energy because of their low cost. The use of space solar power systems and magnesium cycles as energy sources ${ }^{4}$ has been proposed.

The optical properties of Nd/Cr:YAG ceramics are discussed below. Large Nd/Cr:YAG crystals $^{8,9}$ cannot maintain their structure. In contrast, Nd/Cr:YAG ceramics can maintain their crystal structure. The ceramics consist of single Nd/Cr:YAG crystals with the diameter of a few $\mu \mathrm{m}$. A continuum in the absorption spectrum due to $\mathrm{Cr}^{3+}$ ions exists in the ultraviolet to visible regime, and such an absorption spectrum reasonably matches the solar spectrum. The main wavelength of laser oscillation is $1064 \mathrm{~nm}$, which is the same as that of $\mathrm{Nd}: \mathrm{YAG}$. Here, we indicate that the phonon-assisted cross-relaxation increases the optical-optical conversion efficiency from solar light to laser. The lasing system is markedly different from that of a conventional laser. The electrons in the $\mathrm{Cr}$ ions and the phonons work as the energy sources for laser actions. ${ }^{10-13}$ The intensity of pumping light, the density of $\mathrm{Nd}$ ions and temperature are important parameters for generating phonon-assisted cross-relaxation. The effect of this phenomenon is marked when the temperatures of the laser materials are high. ${ }^{12}$

Enhancing the cross-relaxation of the $\mathrm{Nd}$ ions enables us to realize a high conversion efficiency from solar light to laser. ${ }^{12}$ A remarkable increase in laser gain and output laser power have already been observed. ${ }^{12}$ Previously, some reports on the measurement of the fluorescence lifetime of the Nd/Cr:YAG ceramics excited with narrow-band light were published. ${ }^{1-14}$ However, an increase in fluorescence lifetime was not observed as a function of temperature. The increase in fluorescence lifetimes of other laser materials by cross-relaxation has been already observed, ${ }^{16-18}$ but the remarkable increase in fluorescence lifetime of $\mathrm{Nd} / \mathrm{Cr}$ :YAG ceramics has been not observed previously. The fluorescence lifetime of the $\mathrm{Nd} / \mathrm{Cr}: \mathrm{YAG}$ ceramics excited with white light, which depends on the temperature of the ceramics, was measured, and a remarkable increase in fluorescence lifetime, which is proof of the cross-relaxation, was observed. At the same time, the dependences of fluorescence lifetime on temperature and doped $\mathrm{Cr}$ ion density were investigated. Finally, the dependence of output laser energy in a laser oscillator on temperature was clarified.

\section{Experimental}

Here, the Nd/Cr:YAG ceramics were fabricated using powder made by the sol-gel method. ${ }^{1}$ In the experiment to measure fluorescence lifetime, transparent ceramics were used. The density of the doped $\mathrm{Nd}$ ion was $1 \%$, and the doped $\mathrm{Cr}$ ion densities were 0.1 and $3 \%$. Ceramic samples, which were not transparent and made of white powder pressed at a high pressure, were also used in the experiment. The doped Nd ion density was the same as the transparent ceramics, and the doped $\mathrm{Cr}$ ion densities were 0.0, 0.3, 1.0, 2.0, 4.0, and 6.0\%, respectively. The diameter of the ceramic samples was $1 \mathrm{~cm}$, and the dimensions of the transparent ceramics were $2 \times 3 \mathrm{~cm}^{2}$. All samples were $3 \mathrm{~mm}$ thick. The active mirrors 


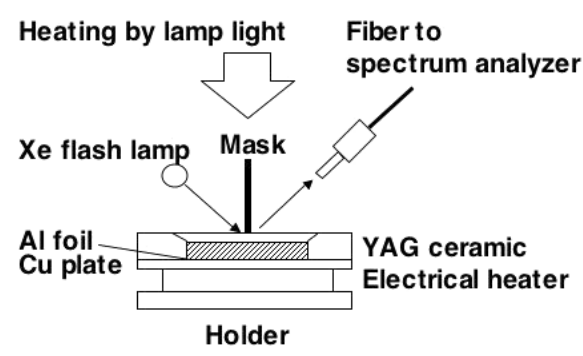

(a)

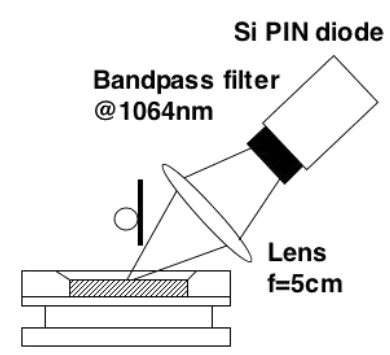

(b)

Fig. 1. Experimental setups. (a) Absorption of lamp light. (b) Fluorescence lifetime of Nd ions.

used $^{20}$ were fabricated from these transparent ceramics. However, it seems that the doped Cr ion density was not controlled accurately, and a difference in absorption coefficient was observed. Experimental setups are shown in Fig. 1. An experimental setup for measuring spectrum of the lamp light through Nd/Cr:YAG ceramics is shown in Fig. 1(a). Firstly, the spectrum was observed to see if the absorbed power of the lamp light changes as a function of temperature. The ceramics were excited optically using a small Xe flash lamp. On the opposite side of the surface of the laser medium, a bulk electrical ceramic heater through an aluminum foil and a copper plate of $3 \mathrm{~mm}$ thickness was set in this experiment. A silicon compound was pasted on the copper plate. An arc-metal-halide lamp was used to heat the ceramic lasers additionally only when the spectrum of the lamp light was observed through Nd/Cr:YAG ceramics. The ceramic could only be heated up to $413 \mathrm{~K}$ by the electrical heater because thermal conduction caused the heat to dissipate from the copper holder. A CW arc lamp was therefore introduced to heat the ceramic up to $473 \mathrm{~K}$. The reflected lamp light was not a problem in this case because the CW lamp was turned off when the transmitted flash lamp light was observed. The temperature of the Nd/Cr:YAG ceramics was controlled and measured using a thermo coupler and a radiation thermometer. A ceramic disk was set on a copper holder, and the temperature difference between the ceramics and copper holder was determined to be below $5 \mathrm{~K}$. The measured temperature of the ceramics is always a few $\mathrm{K}$ lower than that of copper holder. The reflected lamp light was measured using a spectrometer (USB4000: Ocean Photonics) to determine whether the absorption varies with temperature. An experimental setup for observing fluorescence decay is shown in Fig. 1(b). Fluorescence was detected through a lens and a bandpass filter at $1064 \mathrm{~nm}$ with $10 \mathrm{~nm}$ bandwidth (FWHM) using a Si PIN photodiode. The signal of fluorescence decay was observed using an oscilloscope (TDS2022B: Textronics). The decay waveforms of the fluorescence were integrated thirty times to increase the signal to noise ratio (SNR).

\section{Results and Discussion}

\subsection{Absorption of lamp light}

The emission spectra of the Xe flash lamp and the optical transmission spectra of the $\mathrm{Nd} /$ Cr:YAG ceramic disk and aluminum foil are shown in Fig. 2. The measured optical 


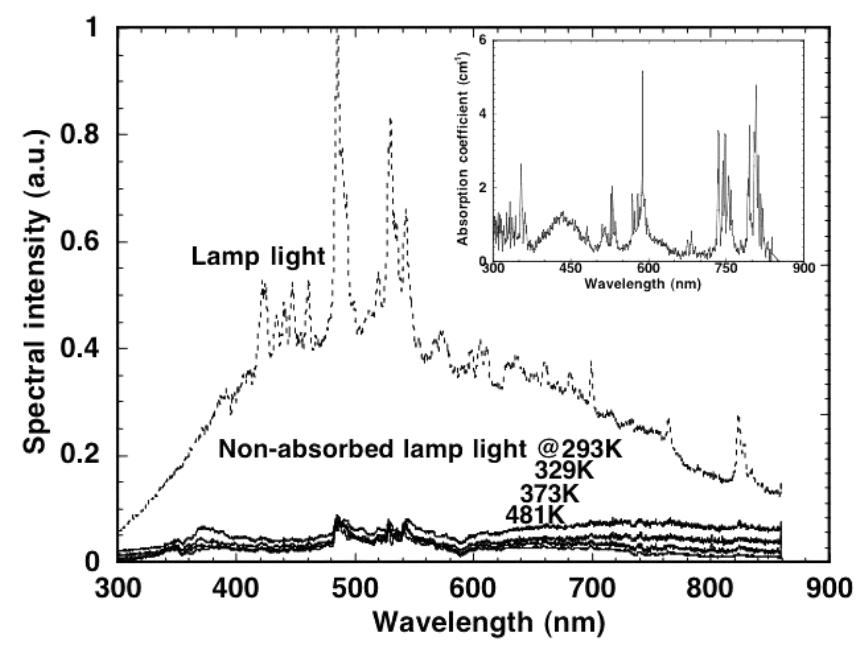

(a)

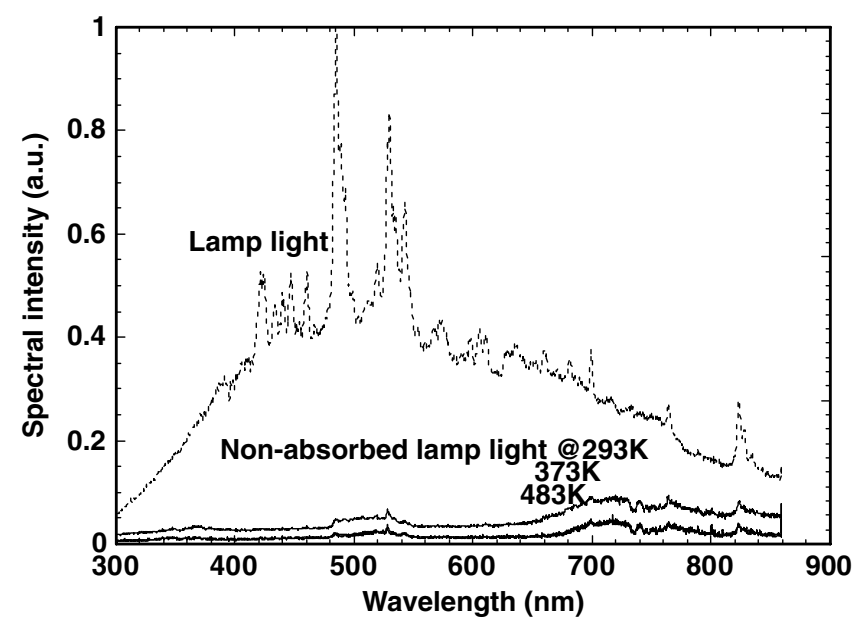

(b)

Fig. 2. Optical transmission spectra of injected lamp light. (a) $0.1 \%$ and (b) $3 \%$ Cr were doped. Absorption coefficient of $0.1 \% \mathrm{Cr}$ doped Nd:YAG ceramic is shown at the upper right in the inset. Dashed lines show the output spectra of the lamp light, and solid lines show the spectra of non-absorbed lamp light through the transparent ceramics. The scale of intensity is the same between (a) and (b). Lines of non-absorbed lamp light for $373 \mathrm{~K}$ and $483 \mathrm{~K}$ in (b) are duplicated.

transmission spectra intensity was lower than the results using quasi-spectrum calculation in particular. The output spectra of the lamp light, whose spectral shape of the absorption is due to Nd ions, was not observed. It was found that the shape of the absorption spectra of the Nd/Cr:YAG ceramics could not be observed and the entire optical transmission spectra was degraded. Also, the absorbed power was not markedly changed by increasing the temperature of the $0.1 \%$ and $3 \%$ Cr-doped YAG ceramics. The absorbed power increased only slightly when the temperature of the ceramics changed from $300 \mathrm{~K}$ to $500 \mathrm{~K}$. 


\subsection{Fluorescence lifetime of $\mathrm{Nd}$ ions}

First, the fluorescence lifetime in the upper level of the Nd ion for the Nd/Cr:YAG ceramics at $291 \mathrm{~K}$ was measured. Its dependence on $\mathrm{Cr}$ ion density is shown in Fig. 3. Here, the capacitance of the condenser was $10 \mu \mathrm{F}$ for exciting the Xe flash lamp (Flash-25R(w)), and the stored electrical energy was $0.2 \mathrm{~J}$. The distance between the flash lamp and the ceramic was set to $0.5 \mathrm{~cm}$. An energy meter (QE12LP-H-MB: Gentech) was used to measure the energy of single lamp light pulse. The estimated irradiation fluence was $16 \mathrm{~mJ} / \mathrm{cm}^{2}$. Also, the pulse duration of the lamplight measured using a Si PIN photodiode was $10 \mu \mathrm{s}$. Thus, the peak intensity was estimated to be $1.6 \mathrm{~kW} / \mathrm{cm}^{2}$. The intensity of the fluorescence was weak and the Si-PIN photodiode could detect components of the flash lamp light with good sensitivity at a wavelength of $1064 \mathrm{~nm}$ whenever the band pass filter was used. However, the electrical signal from the Si-PIN photo diode decayed by $50 \mu$ s after the flash lamp started emitting. This data was not used in the analysis of the decay time. Linear fitting was conducted to evaluate the decay time of the fluorescence. The mask was separated from the copper holder to capture more intense fluorescence, as shown in Fig. 1(b). When observing the transmitted flash lamp light, the mask prevented the Si-PIN photo diode from detecting the direct flash lamp light.

The small circles and large squares show the results for the ceramics made of white powder and transparent ceramics, respectively. The black squares and triangles show the results measured by Nakatsuka and Fujioka et al. ${ }^{1}$ when Nd/Cr:YAG ceramics were excited by 440-nm and 590-nm light. As shown in Fig. 3, a material such as Nd/Cr:YAG or GSGG shows a non-exponential decay and has two decay components. The long component

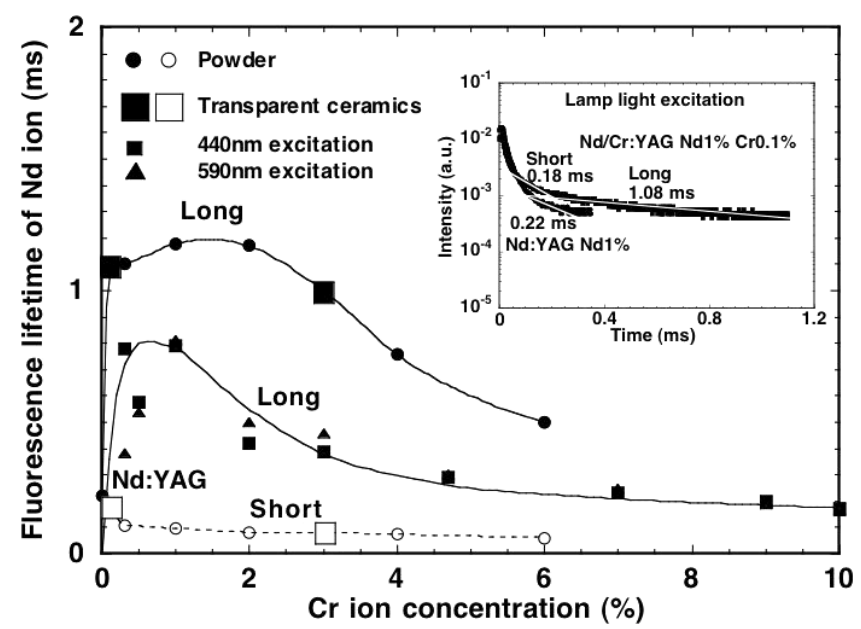

Fig. 3. Evaluated fluorescence lifetime of excitations in Nd/Cr YAG ceramics at $292 \mathrm{~K}$. These ceramics were excited by lamp light. Measured fluorescence decays of Nd:YAG and Nd/Cr YAG ceramics are shown at the upper right in the inset. As shown in this experiment, a material such as Nd/Cr:YAG or GSGG shows a nonexponential decay and has two decay components. The large squares show the results for the transparent ceramics. The black squares and triangles show the results measured by Nakatsuka and Fujioka et al. ${ }^{1}$ when Nd/Cr:YAG ceramics were excited by 440-nm and 590-nm light. 


\section{T. Saiki et al.}

appears after the short one. The short component of below $100 \mu \mathrm{s}$ may occur due to the shortening of coherent time of the $\mathrm{Nd}$ ion affected by the polarization generated in the $\mathrm{Cr}$ ion. The long component are due to the Cr ion transfer energy to Nd ion that occurs slowly over a period of $1 \mathrm{~ms}$. When the Cr ion density was $0.1 \%$, the effective fluorescence lifetime increased to $1.1 \mathrm{~ms}$ owing to the energy transfer from $\mathrm{Cr}$ ions. When the doped $\mathrm{Cr}$ ion density was $3 \%$, the effective fluorescence lifetime was enhanced to $1 \mathrm{~ms}$ due to the changed long energy transfer time $\tau_{F}$ from $\mathrm{Cr}$ ions, too. Some numerical models for the waveform of the fluorescence decay had been already proposed. In the case of the Nd:YAG ceramics, a typical result was obtained. The fluorescence lifetime of the Nd:YAG ceramics was changed from 230 to $60 \mu$ s by increasing their temperature. It decreased and approached a steady value. This is because Nd ions at the ground level were excited to the lower level by heating the ceramics. Nd:YAG ceramics and crystals acts as a 3-level laser system at a high temperature.

The irradiation intensity dependence of the effective fluorescence lifetime for the doped density of $\mathrm{Cr}$ ion of $3 \%$ is shown in Fig. 4. When the irradiation intensity was reduced by changing the distance to $1 \mathrm{~cm}$, the effective fluorescence lifetime increased to $1.2 \mathrm{~ms}$. This occurs due to the amplified spontaneous emission (ASE) effect. We can also mention that the energy transfer from $\mathrm{Cr}$ ion to $\mathrm{Nd}$ ion occurs efficiently.

The measured effective fluorescence lifetimes of Cr-doped Nd:YAG ceramics as a function of temperature are shown in Fig. 5. All the fluorescence lifetimes of Cr-doped Nd:YAG ceramics were increased. Such an increase was evidently observed in the cases of the $1 \%$, and $2 \%$ Cr doped Nd:YAG ceramics. The fluorescence lifetime increased to $1.8 \mathrm{~ms}$, which is the same as that of the $\mathrm{Cr}^{3+}$ :YAG at $293 \mathrm{~K}$. Also, it has been considered that the fluorescence lifetime peaks at $291 \mathrm{~K}$ in the case of the Cr- $3 \%$ doped Nd:YAG. The temperatures at which the fluorescence lifetime peaks vary with the doped density of the $\mathrm{Cr}$ ion and the population

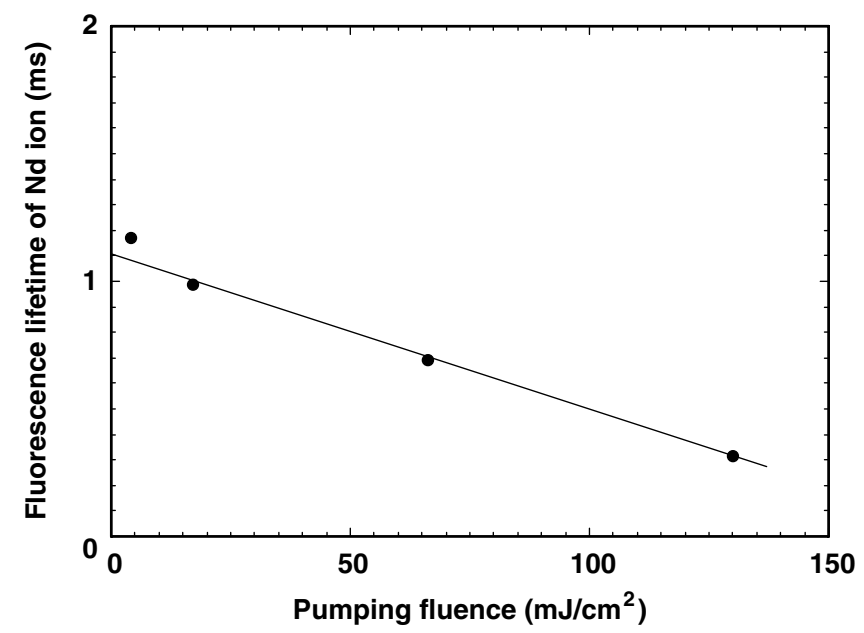

Fig. 4. Degradation of effective fluorescence lifetime for pump fluencies is shown when $3 \%$ Cr was doped. This occurs due to the amplified spontaneous emission (ASE) effect. 


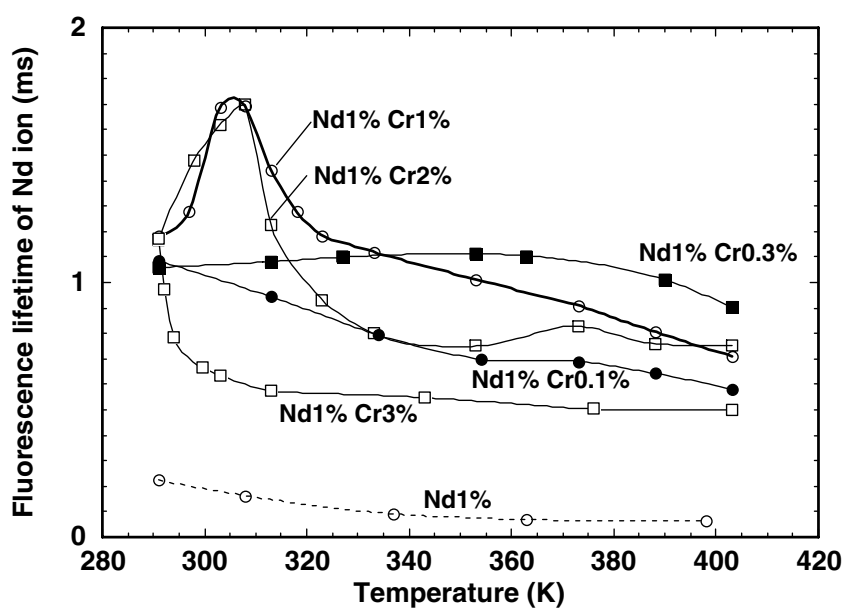

Fig. 5. Evaluated effective fluorescence lifetime as a function of temperature.

number in the lower level of the $\mathrm{Nd}$ ion. When the doped density of the $\mathrm{Cr}$ ion is low, the effective fluorescence lifetime peaks at a high temperature. When the doped density of the $\mathrm{Cr}$ ion is high, the fluorescence lifetime peaks at a low temperature because of the large absorption by the $\mathrm{Cr}$ ion. In particular, it has been found (see Figs. 3 and 5) that the optimum $\mathrm{Cr}$ ion density is $1-2 \%$ when the doped density of the $\mathrm{Nd}$ ion is $1 \%$. At $292 \mathrm{~K}$, when the $\mathrm{Cr}$ ion density is much greater than the $\mathrm{Nd}$ ion density, the long effective fluorescence lifetime decreases.

We introduce a theory for the common increment of effective fluorescence via the crossrelaxation effect. The increase of the fluorescence lifetime has been commonly observed in the Tm:YAG and Cr/Tm:YAG.$^{16-18}$ In these cases, the fluorescence lifetime increased after it decreased following an increase in temperature. It has been thought that the crossrelaxation effect is an energy transfer from excited active ions in the upper level to neighboring active ions in the lower level by multi-phonon relaxation. This energy transfer is conducted in a 2-step non-radiative transition process. (1) At the lasing level, after an active ion absorbs a photon with high energy, it is excited to the pumping level, and the active ion excites another neighboring active ion in the lower level while assisted by the phonons at the same time. (2) The excited active ions at the upper level re-excite the original active ions or additional neighboring ions. This energy transfer occurs by a multi-phonon relaxation. Two simple energy levels (an upper level and a lower level) of active ions in a laser material in a 4-level lasing system are shown in Fig. 6. $N_{1}$ and $N_{2}$ are the population numbers of active ions at the lower and higher levels, respectively. Here, the laser material has a high temperature, and when excitation is not performed, some population numbers exist in the lower level depending on the Boltzmann distribution. In such a case, the laser material acts as a 3-level laser system. We also assumed a condition in which $N_{1}$ is equal to 0 when exciting and after exciting when the fluorescence has vanished. $\tau_{R}$ is the fluorescence lifetime indicated by the transition of active ions from the upper level to the lower level. $\tau_{R 12}$ is the decay time indicated by the excitation of active ions from the lower level to the upper level. 


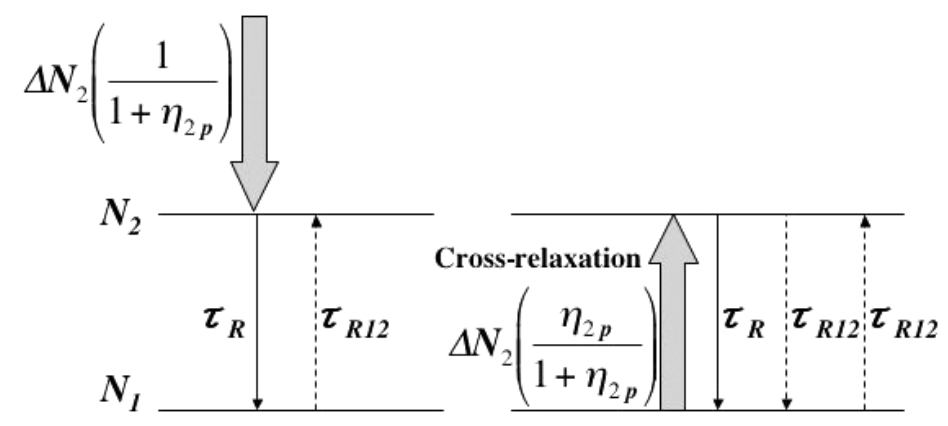

Fig. 6. Cross-relaxation effect at upper level and lower level in a laser system.

We assumed that the relaxation time from the upper level to the lower level was the same as the decay time. $\eta_{2 p}$ is the ratio of the cross-relaxation and set as $0<\eta_{2 p} \leq 1 . \Delta N_{2}$ is the total pump rate in the laser system. Here, the rate equation for $N_{2}$ is given by

$$
\frac{d N_{2}}{d t}=-\frac{N_{2}}{\tau_{R}}+\frac{N_{2}\left(\frac{\eta_{2 p}}{1+\eta_{2 p}}\right)}{\tau_{R 12}}+\Delta N_{2}=-\frac{N_{2}}{1 /\left\{\frac{1}{\tau_{R}}-\frac{\left(\frac{\eta_{2 p}}{1+\eta_{2 p}}\right)}{\tau_{R 12}}\right\}}+\Delta N_{2} .
$$

In the right side of Eq. (1), the first term shows the decay by normal spontaneous emission and the second term shows the re-excitation of the active ions at the lower level due to energy transfer from the excited active ions in the upper level by the cross-relaxation. Here, spontaneous emissions from the excited Nd ions and re-excitations of the neighboring $\mathrm{Nd}$ ions to the upper level occur at the same time. Theoretically, this should result in an increment in the fluorescence lifetime. If the pump rate increases by active ions exciting to the pumping level, there should be no change in the fluorescence lifetime.

From Eq. (1), we obtain an equation for an effective fluorescence lifetime given by

$$
\tau_{R}^{\prime}=1 /\left\{\frac{1}{\tau_{R}}-\frac{\left(\frac{\eta_{2 p}}{1+\eta_{2 p}}\right)}{\tau_{R 12}}\right\} .
$$

When $\eta_{2 p}$ is equal to 1 , it is clear from the experimental results that the effective fluorescence lifetime $\tau_{R}^{\prime}$ is equal to $2 \tau_{R}$. From Eq. (2), we can obtain the relation that $\tau_{R 12}$ is equal to $\tau_{R}$. Thus, Eq. (2) is rewritten simply as

$$
\tau_{R}^{\prime}=\left(1+\eta_{2 p}\right) \cdot \tau_{R}
$$

When $\eta_{2 p}$ is equal to $0, \tau_{R}^{\prime}$ is equal to $\tau_{R}$. In terms of property of the fluorescence decay, when an excitation of the active ions is performed and vanishes, the time $t$ is set to 0 . We then assume that $\Delta N_{2}$ is equal to 0 , and Eq. (1) is rewritten as

$$
\frac{d N_{2}}{d t}=-\frac{N_{2}}{\tau_{R}^{\prime}}
$$


Here, by solving Eq. (4), we obtain an equation for $\mathrm{N}_{2}$, as

$$
N_{2}(t)=N_{2}(0) \cdot e^{-\frac{t}{\tau_{R}^{\prime}}} .
$$

Also, when we assume that $\Delta N_{2}$ is constant and the excitation of the active ions is performed continuously, the equation for $N_{2}$ in the steady state is given by solving Eq. (1) with the condition $\frac{d N_{2}}{d t}=0$, as

$$
N_{2}=\Delta N_{2}\left(1+\eta_{2 p}\right) \cdot \tau_{R}
$$

We find from Eq. (6) that $N_{2}$ when $\eta_{2 p}$ is equal to 1 is twice as large as when $\eta_{2 p}$ is equal to 0 under $\mathrm{CW}$ pumping.

The energy diagram of the $\mathrm{Nd}^{3+} / \mathrm{Cr}^{3+}$ :YAG is shown in Fig. 7 . We will now discuss the status of excited $\mathrm{Cr}$ ions. In the mechanism of absorption and emission from the $\mathrm{Cr}^{3+}$ :YAG crystal, the interaction between the energy levels of excited $\mathrm{Cr}$ ions and phonons in the YAG lattices (which is called electron-phonon coupling) is very important and results in the broadening of the energy level. The phonon energy of Cr ions in the YAG crystal is $500 \mathrm{~cm}^{-1}$. We can see that there are two thermally vibronic levels of ${ }^{4} \mathrm{~T}_{1}$ and ${ }^{4} \mathrm{~T}_{2}$ : these levels connect the phonons. At an energy level of $20000 \mathrm{~cm}^{-1}$, the two energy levels are tightly coupled. A rapid energy transfer (of less than picoseconds) occurs due to phononassisted dipole-dipole interaction. It is not possible to excite $\mathrm{Cr}$ ions from ${ }^{2} \mathrm{E}$ level to ${ }^{4} \mathrm{~T}_{1}$ level at $500 \mathrm{~K}$ because an energy gap of $5000 \mathrm{~cm}^{-1}$ between the ${ }^{2} \mathrm{E}$ and ${ }^{4} \mathrm{~T}_{1}$ levels exists. The vibration of the $\mathrm{Cr}$ ions due to phonons couples with the electrons and generates polarization. After the absorption of pumped solar light, $\mathrm{Cr}$ ions are excited at the upper level of ${ }^{2} \mathrm{E}$. The $\mathrm{Cr}$ ions are excited from the ${ }^{2} \mathrm{E}$ level to two broad vibronic levels thermally. ${ }^{10}$

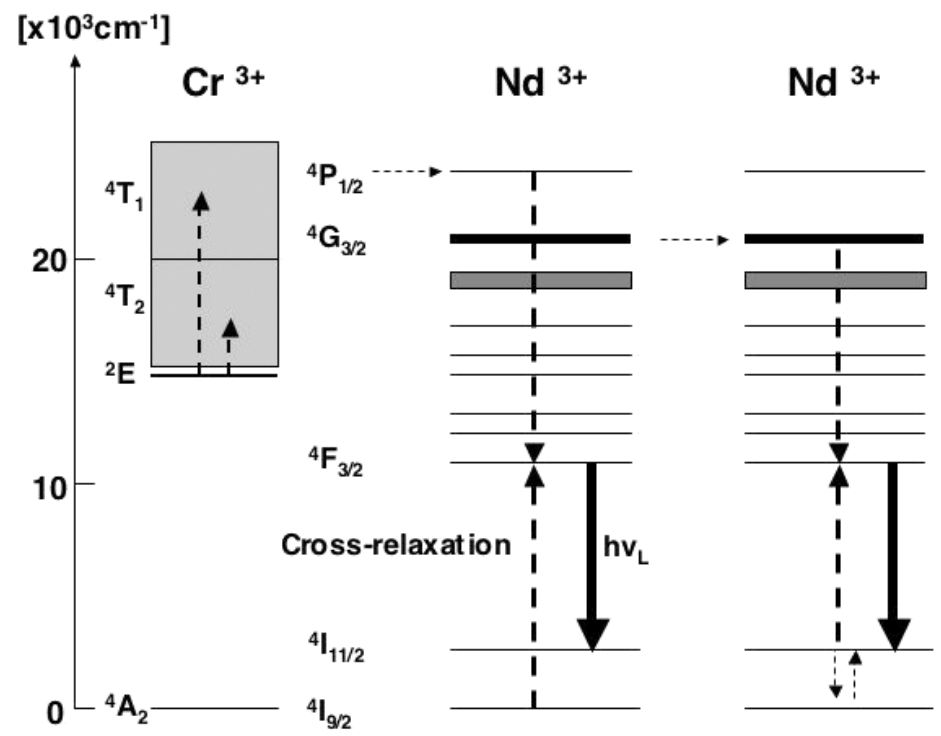

Fig. 7. Energy diagram of $\mathrm{Nd}^{3+} / \mathrm{Cr}^{3+}: \mathrm{YAG}$. The cross-relaxation needs assistance from thermal energy owing to the large energy gap between the ground level and the ${ }^{4} \mathrm{G}_{3 / 2}$ or ${ }^{4} \mathrm{P}_{1 / 2}$ level. 
Cr ions at the two vibronic levels excite the $\mathrm{Nd}$ ions at the ground level. There is an energy gap of $800 \mathrm{~cm}^{-1}$ between the ${ }^{2} \mathrm{E}$ and ${ }^{4} \mathrm{~T}_{2}$ levels at $77 \mathrm{~K}$. At more than $293 \mathrm{~K}$, the thermal excitation of the population occurs from the ${ }^{2} \mathrm{E}$ level to the ${ }^{4} \mathrm{~T}_{2}$ level. Alexandrite lasers have the same pumping system. Fluorescence properties are observed when the Cr ions at ${ }^{4} \mathrm{~T}_{1}$ and ${ }^{4} \mathrm{~T}_{2}$ decay to the ground level by thermal pumping at more than $293 \mathrm{~K} .{ }^{10}$ The spectral peaks shift to the long wavelength side due to the Stokes shift. The spectral peak of the fluorescence emitted from the ${ }^{4} \mathrm{~T}_{1}$ level is $695 \mathrm{~nm}$ and that from the ${ }^{4} \mathrm{~T}_{2}$ level is $720 \mathrm{~nm} .{ }^{10}$ As a result of the analysis, we found that the integrated spectral intensity of the fluorescence components from ${ }^{4} \mathrm{~T}_{2}$ was equal to that from ${ }^{4} \mathrm{~T}_{1}$. This means that the population density in the ${ }^{4} \mathrm{~T}_{2}$ level is the same as that in the ${ }^{4} \mathrm{~T}_{1}$ level. The thermally pumped Cr ions in the two bands strongly contribute to excitation of $\mathrm{Nd}$ ions in the ground level.

We considered a model for calculating the population densities of $\mathrm{Cr}$ and $\mathrm{Nd}$ ions with $\mathrm{CW}$ solar-light pumping. ${ }^{11}$ Firstly, $\mathrm{Cr}$ ions are excited thermally from ${ }^{2} \mathrm{E}$ level to the ${ }^{4} \mathrm{~T}_{1}$ and ${ }^{4} \mathrm{~T}_{2}$ levels after lamp light excitation. The energy transfer from the ${ }^{4} \mathrm{~T}_{1}$ and ${ }^{4} \mathrm{~T}_{2}$ levels of Cr ions to $\mathrm{Nd}$ ions by phonon-coupled electron-electron dipole occurs. ${ }^{10} \mathrm{The}^{4} \mathrm{~T}_{1}$ and ${ }^{4} \mathrm{~T}_{2}$ levels overlap, and the energy transfer from the ${ }^{4} \mathrm{~T}_{2}$ level to ${ }^{4} \mathrm{~T}_{1}$ level occurs efficiently due to multi-phonon relaxation. ${ }^{10,12,13}$ The first process is indicated in Fig. 7, which is independent of the temperature of the laser medium. In the transition from the ${ }^{4} \mathrm{P}_{1 / 2}$ and ${ }^{4} \mathrm{I}_{9 / 2}$ levels to the ${ }^{4} \mathrm{~F}_{3 / 2}$ level, cross-relaxation due to multi-phonon relaxation occurs. ${ }^{12,15,19}$ The second process is important. It induces much excitation of $\mathrm{Nd}$ ions at the lower level by crossrelaxation and depends on the temperature of the laser medium. Additionally, in the transition from the ${ }^{4} \mathrm{G}_{3 / 2}$ and ${ }^{4} \mathrm{I}_{11 / 2}$ levels to the ${ }^{4} \mathrm{~F}_{3 / 2}$ level, cross-relaxation occurs. The energy gap between the ground level and the ${ }^{4} \mathrm{G}_{3 / 2}$ level is $20700 \mathrm{~cm}^{-1}$. The photon energy at the wavelength of $1064 \mathrm{~nm}$ is $9400 \mathrm{~cm}^{-1}$.

From Eq. (6), an increase of the fluorescence lifetime means an increase of the pump rate. Thus, the measured effective fluorescence lifetime is proportional to the ratio of the increase for the inverse population density. Thus, the equation for the effective fluorescence lifetime of $\mathrm{Nd}$ ions, $\tau_{N d}^{\prime}$, for the $\mathrm{Nd} / \mathrm{Cr}$ :YAG ceramics is given $\mathrm{as}^{12}$

$$
\tau_{N d}^{\prime}=\left(\frac{N_{2}-N_{L} / n}{N_{20}}\right) \cdot \tau_{N d 0}^{\prime},
$$

where $N_{2}$ is the inverse population density, and $N_{20}$ is the inverse population density at $292 \mathrm{~K}$. The population density increases with the temperature of the laser medium. $N_{L}$ is the population in the lower level of the $\mathrm{Nd}$ ions. $n$ is the split number of the ${ }^{4} \mathrm{~F}_{3 / 2}$ level, which we set to $6 . N_{2}$ is expressed as

$$
N_{2}=\left[\Delta N_{C r}\left(1+\eta_{2 p}\right)+\Delta N_{N d}\right] \cdot \tau_{N d 0}^{\prime} .
$$

Here, $\Delta N_{C r}$ is the pump rate of Cr ions, $\Delta N_{N d}$ is the pump rate of $\mathrm{Nd}$ ions, and $\tau_{N d 0}^{\prime}$ is the effective fluorescence lifetime of the upper level of $\mathrm{Nd}$ ions at $291 \mathrm{~K}$. The structure of Eq. (7) is almost the same as that of Eq. (3) because only $1+\eta_{2 p}$ was multiplied to the effective fluorescence lifetime $\tau_{N d}^{\prime}$ when neglecting $\Delta N_{N d}$ and $N_{L}$. When the $\eta_{2 p}$ is equal to 1.0 in Eq. (8) and the pump rate increased by a factor two, then the population inversion increase 
by a factor of two and the fluorescence lifetime increases close to two. In fact, the fluorescence lifetime increased from 1.1 to $1.8 \mathrm{~ms}$ when $1 \% \mathrm{Cr}$ were doped in this experiment. We have obtained a good agreement between the experiment and theory. Whenever the doped Cr ion density was $0.1 \%$, and the temperature of the ceramics increased, then the lifetime and small signal gain increased. This is caused by the phonon-assisted cross-relaxation discussed previously. ${ }^{12}$ The cross-relaxation in $\mathrm{Nd}$ ions needs assistance from thermal energy owing to the large energy gap between the ground level and the ${ }^{4} \mathrm{G}_{3 / 2}$ or ${ }^{4} \mathrm{P}_{1 / 2}$ level. It has been proved that the speed of the energy transfer is unit whenever the temperature of the laser media changes. The speed is only decided by the distance between $\mathrm{Cr}$ ion and $\mathrm{Nd}$ ion. If the speed changes faster, the effective fluorescence lifetime should degrade. Enhancement of the output laser energy and the effective fluorescence lifetime could be explained only by the cross-relaxation effect in the energy transfer process between ${ }^{4} \mathrm{~T}_{1}$ level of $\mathrm{Cr}$ ion to the energy level of $\mathrm{Nd}$ ion. The increase in the absorption of the lamp light does not affect the lifetime of the Nd/Cr:YAG ceramics. The upconversion process in the level of the doped $\mathrm{Nd}$ ion is neglectable, and it has not been considered that these phenomena affect effective fluorescence lifetimes of laser gains.

The change of the fluorescence spectral shape as a function of temperature were not observed in these experiments. Thus, it has been shown that the effective stimulated emission cross-section remained intact from 300 to $500 \mathrm{~K} .{ }^{15}$

\subsection{Laser oscillation}

We examined a Nd/Cr:YAG ceramic laser employing a flash lamp ${ }^{21}$ with the temperature of the laser material controlled. The experimental setup is shown in Fig. 8. The laser oscillator consists of single flash lamp. The input electrical energy was $20 \mathrm{~J}$. The pulse duration of the

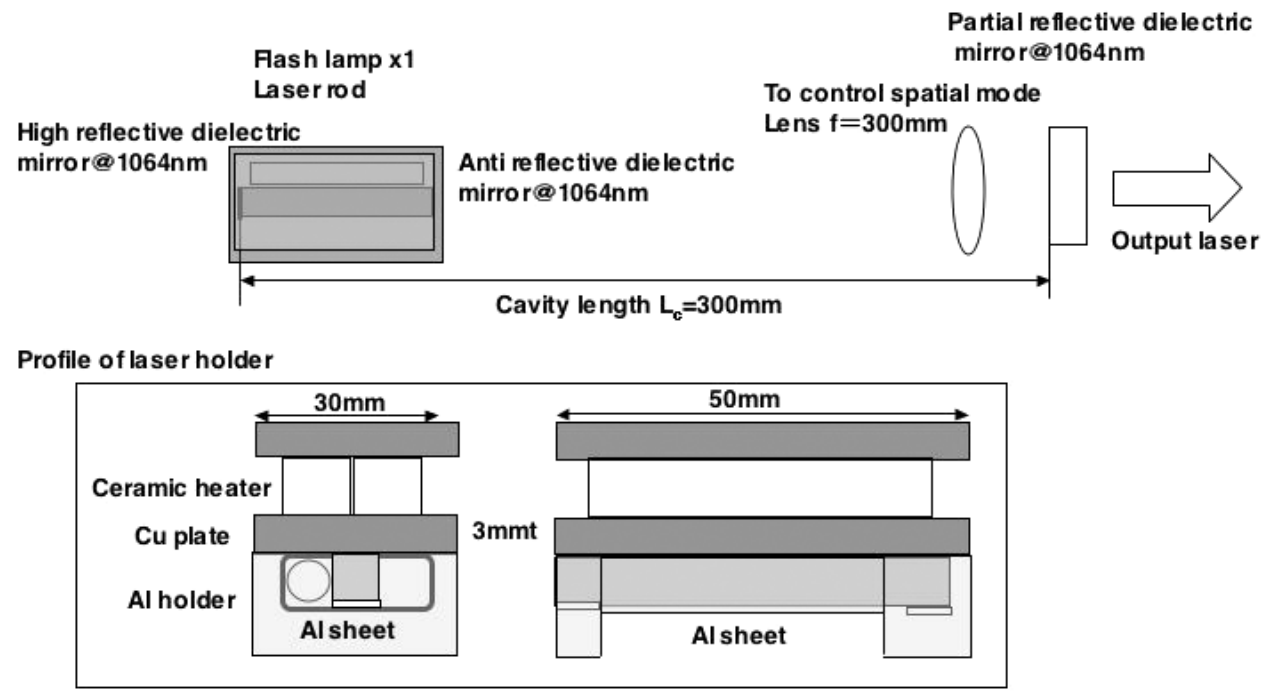

Fig. 8. Experimental setup for laser oscillation. 
lamplight was $120 \mu \mathrm{s}$. The calculated irradiated intensity of the Xe lamp light on the ceramic was $1.4 \mathrm{~kW} / \mathrm{cm}^{2}$. The capacitance of the capacitor used was $210 \mu \mathrm{F}$ in the flash-lamp circuit. The diameter of the flash-lamp tube used is $3.5 \mathrm{~mm}$ and the length is $40 \mathrm{~mm}$. The lamp tube is covered with a Teflon tube to limit the light components in the violet regime. Pump light is injected into the side surface of the Nd/Cr:YAG ceramic rod (Kohnoshima Chemical). The profile of the ceramic rod is square and the size of the laser rod is $4 \times 4 \times 48 \mathrm{~mm}^{3}$. The Nd density and the Cr density of the ceramic rod was $1.0 \%$ and $0.1 \%$, respectively. A configuration of flat-concave cavity was used here. Thus, all the stored energy in the laser rod should be consumed. The cavity length was set at $30 \mathrm{~cm}$ to control the divergence of the laser beam.

An energy meter (QE12LP-H-MB: Gentech) was used to measure laser energy by a single shot. The electrical signals were monitored using an oscilloscope. The reflectivity of the output mirror was $90 \%$. A laser oscillation by free-running modes was performed in the experiment. The aluminum laser holder was set under a copper plate and the laser rod was fixed directly on the copper plate. Ceramic heaters were set on the plate and the plate was heated. The size of single ceramic heater is $10 \times 10 \times 40 \mathrm{~mm}^{2}$.

The electrical input power into the ceramic heaters was controlled by a power supply. The distribution of the temperature for the laser rod should be almost uniform because the thickness was $4 \mathrm{~mm}$, and the laser rod was fixed directly as well as enclosed in an aluminum foil. The temperature of the copper plate was measured using a thermo meter (TN006: OHM), and the temperature of the laser rod was decided. An analysis for the population inversion density had been also performed. The output laser power of the laser oscillator was given by ${ }^{22}$

$$
\frac{E_{\text {out }}}{\Delta t_{p}} \propto I_{s}^{\prime} \cdot\left[\frac{2 \sigma_{s}^{\prime} \Delta N \cdot l}{2 \alpha_{0} l-\ln \left(R_{o c}\right)}-1\right] .
$$

Here, $I_{s}^{\prime}$ is the effective saturation power, $E_{\text {out }}$ is the output laser energy, $\Delta t_{p}$ is the duration of the laser pulse, $R_{o c}$ is the reflectivity of the output coupler, $\sigma_{s}^{\prime}$ is the effective stimulated emission cross-section, $l$ is the length of the laser rod and $\alpha_{0}$ is the loss coefficient of the laser rod. An equation for the normalized inversed population density was introduced from Eq. (9). The normalized inversed population density is given by

$$
x=\frac{\Delta N \cdot l}{\Delta N_{p} \cdot l} \simeq \frac{E_{\text {out }} / \Delta t_{p}}{E_{\text {outp }} / \Delta t_{p}}=\frac{E_{\text {out }}}{E_{\text {outp }}} .
$$

Here, $\Delta N$ is the inversed population density at the peak time of the pulse duration, $\Delta N_{p}$ is the maximum one, and $E_{\text {out }}$ is the maximum output laser energy at the peak. Thus, Eq. (10) shows that $x$ is decided by the ratio of $E_{\text {out }} / E_{\text {outp }}$. An equation for $\eta_{2 p}$ was introduced from Eq. (8). $\eta_{2 p}$ is given by

$$
\eta_{2 p}=(2 x-1)+(x-1) \cdot \frac{\Delta N_{N d}}{\Delta N_{C r}} .
$$

Here, $\Delta N_{N d} / \Delta N_{C r}$ was set to be $1 / 5 \cdot{ }^{11,12}$ However, Eq. (11) can be used only when the output laser energy increases. After the output laser energy peaks, $\eta_{2 p}$ is set to 1 . 


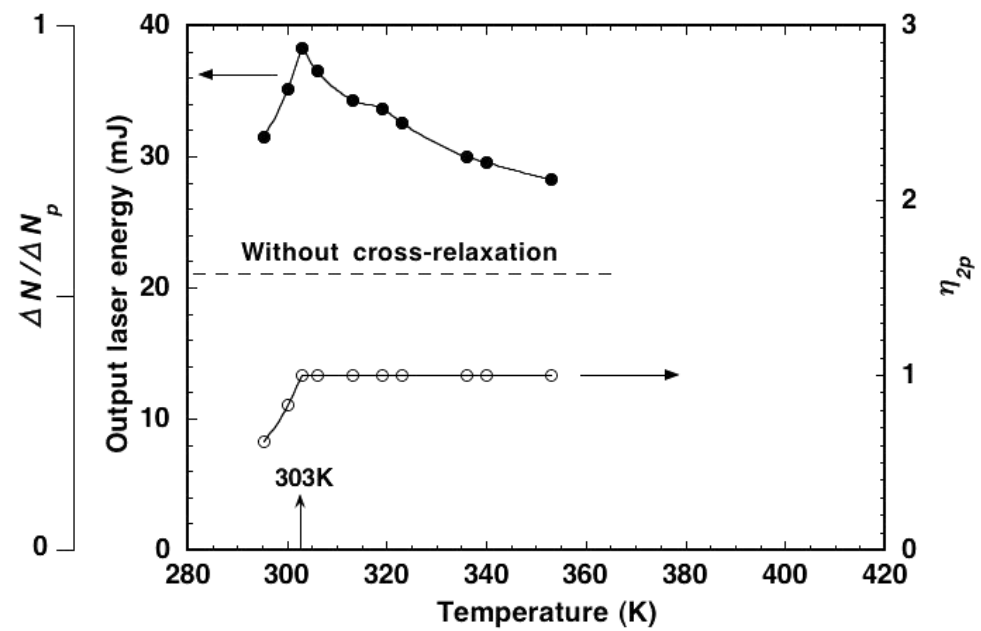

Fig. 9. Measured output laser energy, evaluated normalized inverse population density and ratio of the crossrelaxation as a function of temperature.

The measured output laser power of the Nd/Cr:YAG ceramic laser, normalized inverse population density, and ratio of the cross-relaxation are shown in Fig. 9. The normalized inverse population density and the ratio of the cross-relaxation were calculated by using Eqs. (10) and (11), respectively. The dashed line shows the output laser energy without cross-relaxation. The maximum output laser power of $39 \mathrm{~mJ}$ was obtained when the temperature of the ceramic was $303 \mathrm{~K}$. Beam profile of the output laser pulse was square. The beam pattern and the divergence angle have not been changed by increasing the temperature of the laser rod. It was near twice the case without cross-relaxation. The output laser energy increased with increasing the temperature. After the output laser energy peaked, it deceased. It had been found that the temperature at which the effective fluorescence peaks is consistent with one at which the output laser energy peaks. $\eta_{2 p}$ was calculated to be 0.6 using Eq. (11) at $290 \mathrm{~K}$, and 1.0 at $400 \mathrm{~K}$.

The peak temperature of the obtained output laser energy was lower than the case of measured effective fluorescence lifetime when the doped density of $\mathrm{Cr}$ ion was $0.1 \%$. The cause is that the pulse duration of the pumped lamp light was $120 \mu$ s and single order longer than that used for measuring fluorescence lifetime. Thus, it means that the thermal load and the energy of the lamp light absorbed by the $\mathrm{Cr}$ ion is a single order higher than the case of this experiment. The same effect was also observed in the case of the estimated effective fluorescence lifetime when the doped density of $\mathrm{Cr}$ ion is high as shown in Fig. 5 .

Measured laser gains of $\mathrm{Nd} / \mathrm{Cr}$ :YAG ceramic laser peaked at $450 \mathrm{~K}$ owing to the cross-relaxation shown in Ref. 23. This is because the irradiated intensity of the CW lamp light on the ceramic was $27 \mathrm{~W} / \mathrm{cm}^{2}$ and was two orders lower than that used in this experiment. Thus, it means that the thermal load per unit time is two orders lower than the case of this experiment. Moreover, the dependence of the laser gains on temperature is almost consistent with the case of the output laser energy. 
In this experiment, for laser oscillation, the electrical energy added to the flash lamp was $20 \mathrm{~J}$ and the converted optical energy was estimated to be $3.2 \mathrm{~J}$ because the evaluated electro-optical conversion efficiency was $16 \%{ }^{21}$ Thus, the maximum opt.-opt. conversion efficiency from flash lamp light to laser was $1.3 \%$ due to the cross-relaxation effect. Without the cross-relaxation effect, it would be $0.66 \%$. Reducing the reflectivity of the output coupler will improve the output energy and the increment of the output laser energy.

Electrical heating was used to enhance the cross-relaxation effect caused by increasing the temperature of the laser material. In other words, we wanted to see if we could accelerate the effect. In an actual laser system, it is easy to heat the laser material to a high temperature by optical excitation owing to the generated heat and the cutting of the heat conduction. In the cross-relaxation process, the heat is transformed into optical energy. This is where the energy balance between the thermal and optical energy is kept. On the basis of the principle of cross-relaxation, half of the output laser energy was converted from thermal energy at the maximum. In the case shown in Fig. 9, the electrical input power of the heater was $2 \mathrm{~W}$, the temperature was $303 \mathrm{~K}$, and the measured output laser energy was $39 \mathrm{~mJ}$. We predicted that $19 \mathrm{~mJ}$ of the thermal energy would be converted to laser energy due to the cross-relaxation effect. However, it is impossible to evaluate either the ratio of heat generated by a heater and that generated by optical excitation, or that which was converted to laser energy. Results showed that the conversion efficiency from the input optical energy and the input thermal energy to the laser energy was very small.

Special attention is required if the limits of a rigid opt-opt. conversion efficiency from lamp light to laser is evaluated. Heat in the laser materials must be perfectly insulated against the outside when laser generation experiments are performed.

\section{Conclusion}

The fluorescence lifetimes of $\mathrm{Nd} / \mathrm{Cr}$ :YAG ceramics were measured to determine whether phonon-assisted cross-relaxation occurs. In this experiment, an increase of the effective fluorescence lifetime was observed. A long lifetime of $1.1 \mathrm{~ms}$ was observed owing to the $\mathrm{Cr}$ ions excited at a temperature of $300 \mathrm{~K}$ whenever the doped $\mathrm{Cr}$ ion density was low. When the temperature of the ceramics was $305 \mathrm{~K}$, the effective fluorescence lifetime increased to $1.8 \mathrm{~ms}$ by the cross-relaxation.

Laser oscillations with the temperature of the laser material controlled were observed. A remarkable increase of the output laser energy was recognized with increasing temperature. The observed maximum output laser energy was close to twice that of the case without cross-relaxation. It has been recognized that the dependence of the output laser energy on temperature is almost consistent with the case of these effective fluorescence lifetime. It has been shown that, at the energy level of the $\mathrm{Nd} / \mathrm{Cr}$ :YAG, the mechanism of the phonon-assisted cross-relaxation exists. 


\section{Acknowledgments}

This research was supported by JAXA, whom we thank for their support. The Nd/Cr:YAG ceramics were supplied by Drs. T. Yanagitani and H. Yagi of Konoshima Chemical Co., Ltd.

\section{References}

1. M. Nakatsuka, K. Fujioka, H. Yoshida and H. Fujita, J. Korean Phys. Soc. 43 (2003) 607.

2. T. Saiki, K. Imasaki, S. Motokoshi, C. Yamanaka, H. Fujita, M. Nakatsuka and Y. Izawa, Opt. Commun. 268 (2006) 155.

3. T. Saiki, S. Motokoshi, K. Imasaki, H. Fujita, M. Nakatsuka and C. Yamanaka, Jpn. J. Appl. Phys. 46 (2007) 156.

4. T. Yabe et al., Appl. Phys. Lett. 90 (2007) 261120.

5. H. Yagi, T. Yanagitani, H. Yoshida, M. Nakatsuka and K. Ueda, Jpn. J. Appl. Phys. 45 (2006) 133.

6. C. G. Young, Appl. Opt. 5 (1966) 993.

7. M. Weksler and J. Shwartz, IEEE J. Quantum. Elect. 24 (1988) 1222.

8. Z. J. Kiss and R. C. Duncan, Appl. Phys. Lett. 5 (1964) 200.

9. W. Koechner, Solid-Level Laser Engineering (Springer-Verlag, New York, 2006), p. 64.

10. P. Hong, X. X. Zhang, C. W. Struck and B. Di Bartolo, J. Appl. Phys. 78 (1995) 4659.

11. T. Saiki, S. Motokoshi, K. Imasaki, K. Fujioka, H. Fujita, M. Nakatsuka, Y. Izawa and C. Yamanaka, Jpn. J. Appl. Phys. 47 (2008) 7896.

12. T. Saiki, M. Nakatsuka and K. Imasaki, Jpn. J. App. Phys. 49 (2010) 082702-1.

13. C. W. Struck and W. H. Fonger, J. Lumin. 10 (1975) 1.

14. M. Yamaga, Y. Oda, N. Kashiwagura, K. Hasegawa, H. Ito and S. Mizuno, Proceeding on the conference of CLEO Europe EQEC 2009.

15. T. Saiki, K. Funahashi, S. Motokoshi, K. Imasaki, K. Fujioka, H. Fujita, M. Nakatsuka, Y. Izawa and C. Yamanaka, Opt. Commun. 282 (2009) 614.

16. G. Huber, E. W. Duczynski and K. Peterman, IEEE J. Quntum. Electron 24 (1988) 920.

17. G. Armagan, A. M. Buoncristiani and B. Di Bartolo, Opt. Mater. 1 (1992) 11.

18. G. Armagan, A. M. Buoncristiani and B. Di Bartolo, J. Lumin. 44 (1989) 129.

19. V. Lupei, Opt. Mater. 16 (2001) 137.

20. J. H. Kelly, D. L. Smith, J. C. Lee, S. D. Jacobs, D. J. Smith, J. C. Lambropoulos and M. J. Shoup III, Opt. Lett. 12 (1987) 996.

21. T. Saiki, S. Motokoshi, K. Imasaki, K. Fujioka, H. Yoshida, H. Fujita, M. Nakatsuka and C. Yamanaka, Jpn. J. Appl. Phys. 48 (2009) 122501-1.

22. W. Koechner, Solid-Level Laser Engineering (Springer-Verlag, New York, 2006), p. 119.

23. T. Saiki, M. Nakatsuka, K. Fujioka, S. Motokoshi and K. Imasaki, Opt. Commun. 284 (2011) 2980 . 